\title{
Structure du peuplement des Harpacticides dans le milieu hyporhéique d'un ruisseau des Pyrénées
}

\author{
R. Rouch1
}

Mots clés : Milieu hyporhėique, Harpacticides, peuplement, conditions hydrodynamiques.

Le peuplement des Harpacticides du milieu hyporhéique du ruisseau de Lachein (Pyrénées) a été étudié pendant un an sur un site de $75 \mathrm{~m}^{2} .21$ espèces dont 9 stygobies ont èté recensées. Ce peuplement est caractérisé par sa stabilité dans le temps puisqu'aucune variation saisonnière importante n'a pu être mise en évidence entre les sept séries de prélèvements réalisćes. En revanche, ce peuplement présente une forte hétérogénéité spatiale : quatre groupements ont été reconnus dont la répartition se superpose aux différentes unités physiographiques définies par les écoulements de surface : bancs de graviers, chenal, zone d'eau morte. Les bancs de graviers constituent le milieu de vie préférentiel des Harpacticides : à cet endroit, les formes hypogées dominent les espéces épigées qui sont repoussées à la périphérie des bancs. Les conditions hydrodynamiques au sein du milieu hyporhéique permettent d'expliquer ce type de distribution. En effet, ce sont les zones oủ la perméabilité est la plus élevée qui abritent les populations les plus abondantes et les plus riches en espèces. Toutefois, ces zones ont une diversité spécifique plus faible en raison des phénomènes de compétition inter-spécifique.

\section{Harpacticoid assemblages from a Pyrenean creek hyporheos}

Keywords : Hyporheos, harpacticoids, assemblage, hydrodynamic conditions.

The hyporheic harpacticoids from a $75 \mathrm{~m}^{2}$ area along Lachein Creek, (French Pyrenees), were studied during one year. Seven bi-monthly collections were sampled in the ten quadrats from this area. Twenty one species with nine stygobionts were collected. This assemblage is characterized by temporal stability : no seasonal variations in relative abundance of the main species were detected between the seven collections. Yet, this assemblage has a large spatial heterogeneity. Four groups have been recognized and their dist ributions are a function of the physiographic environments within the stream channel : two gravel bars, a channel and a stagnant water zone. The gravel bars constitute the favoured environment for harpacticoids. Here, stygobiont species are more abundant than epigeous species. The epigeous species are more important along the periphery of the gravel bars. Hydrodynamic conditions in the hyporheal zone explain this distribution. The most abundant populations with the greatest species richness occur where stream bed permeabilities are greatest. However, at this place, the diversity is lower because of competitive exclusion.

Les Harpacticides représentent un des groupes les plus caractéristiques de la faune hyporhéique. Depuis le travail intial de Chapuis (1936), le nombre d'espèces stygobies découvertes dans ce milieu n'a cessé de s'accroître (Rouch 1986), donnant lieu à une très importante littérature tant sur les plans taxonomique que faunistique. Cependant, bien que les recherches écologiques sur le milieu hyporhéique aient connu un réel essor au cours de la dernière

1. Laboratoire souterrain du C.N.R.S., Moulis, 09200 SaintGirons, France. décennie (Danielopol 1989, 1991), peu de travaux, menés à un niveau d'échelle métrique ou décamétrique, ont été consacrés à l'étude de la structure et de l'évolution des peuplements d'Harpacticides (Whitman \& Clark 1984, Pennak \& Ward 1986, Kowarc 1986, 1987, 1990 a et b, 1991, Strayer 1988).

Dans les sédiments du ruisseau de Lachein (Ariège, France), les Harpacticides constituent le groupe de Crustacés le plus diversifié et le plus abondant (Rouch 1988). Sur le site étudié, le rang des espèces selon leur dominance et leur fréquence, de même que les densités, ne présentent pas de 
différences significatives au fil des saisons. En revanche, la distribution spatiale de ces densités, celle du pourcentage des formes hypogées dans les échantillons ainsi que la répartition de certaines espèces, témoignent d'une importante hétérogénéité (Rouch op. cit.).

Le présent travail a pour but de tester les deux hypothèses suivantes : existe-t-il ou non une variation saisonnière de ce peuplement d'Harpacticides? L'hétérogénéité spatiale observée se traduit-elle par des structures de peuplement différentes ?

\section{Site étudié, méthodes utilisées}

Le ruisseau de Lachein, affluent du Lez, est alimenté, pour l'essentiel, par les exutoires du système karstique du Baget (Rouch 1988). Son régime est de type pluvial océanique avec des hautes eaux hivernales et printanières et des basses eaux estivales et automnales; son débit moyen est de $0,523 \mathrm{~m}^{3} / \mathrm{s}$.

La zone étudiée, située à $830 \mathrm{~m}$ en aval de la source, correspond à une aire de $75 \mathrm{~m}^{2}$, arbitrairement divisée en 10 secteurs pour faciliter le repérage des prélèvements (fig. 1). Sur ce site, les écoulements de surface déterminent quatre zones principales : deux bancs de graviers, l'un en rive gauche (secteurs J, H, F et une partie de D), l'autre en rive droite (secteur A et une partie de C) ; un chenal (secteurs I, G, E et une partie de C) ; une zone d'eau morte (secteur B et une partie de D). Ces zones diffèrent par leur granulométrie, leur porosité (Rouch 1988) de même que par les caractéristiques chimiques de l'eau interstitielle (Rouch et al. 1989). Le secteur situé sous la zone d'eau morte se distingue notamment de tous les autres par ses faibles teneurs en $\mathrm{O}_{2}$ dissous $(16,2 \%$ en moyenne).

Tous les deux mois, pendant un an (septembre 1985 - septembre 1986), 20 prélèvements ( 2 par secteurs) de 10 litres chacun ont été réalisés à une profondeur moyenne de $60 \mathrm{~cm}$ par la méthode des sondages tubés (Bou et Rouch 1967). Au total, 130 échantillons ont été prélevés, la série de contrôle de septembre 1986 ne comportant que 10 prélevements. Ces échantillons sont caractérisés par la lettre du secteur dont ils sont issus $(A, B, C, \ldots J)$ et par un numéro : $1-2$ (septembre 1985), 3-4 (novembre 1985), 5-6 (février 1986), 7-8 (mars 1986), 9-10 (mai 1986), 11-12 (juillet 1986), 13 (septembre 1986). Chacun d'entre eux, tamisé sur un filęt dę $100 \mu^{\mathrm{m}} \mathrm{de}$

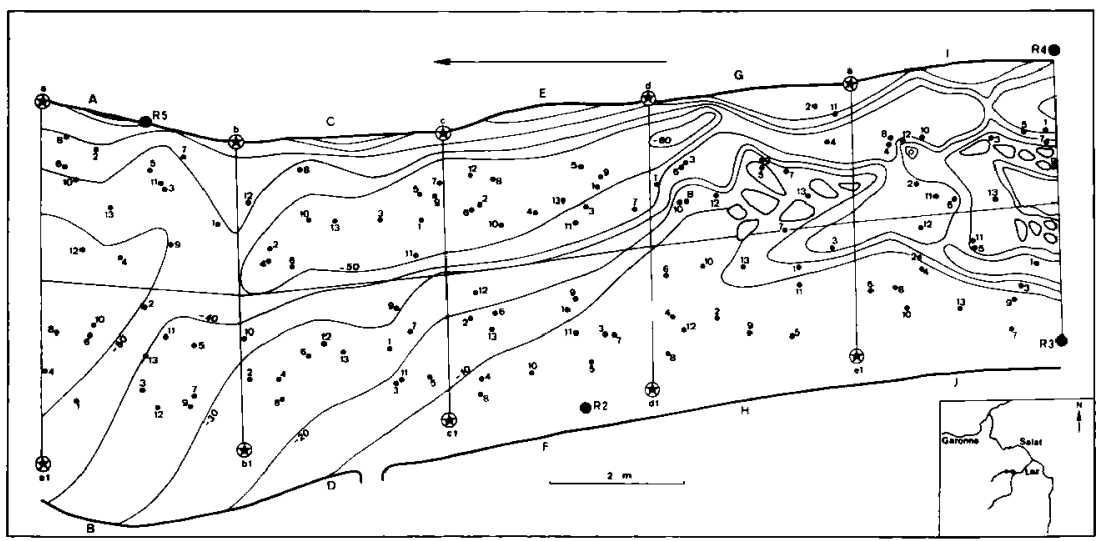

Fig. 1. Carte du site étudié avec l'emplacement des 130 prèlèvements. Les lignes de niveau $(-10,-20$, etc.) correspondent aux profondeurs mesurées en $\mathrm{cm}$ pendant l'étiage $(80 \mathrm{l} / \mathrm{s})$. 
maille, a été fixé dans l'alcool à $70^{\circ}$ puis, après coloration au rose bengale, trié dans sa totalité à la loupe binoculaire. Les déterminations et les dénombrements d'Harpacticides ne prennent en compte que les adutes et les stades copépodites.

\section{Richesse spécifique}

Les 21 espèces recensées sur le site appartiennent à quatre familles : les Phyllognathopodidae, les Ameiridae, les Canthocamptidae et les Parastenocarididae (Tableau I). 9 de ces espèces sont stygobies : Parapseudoleptomesochra subterranea, Nitocrella gracilis, Moraria catalana, Ceuthonectes gatlicus, Elaphoidella bouilloni, Elaphoidella coiffaiti, Antrocamptus chappuisi, Parastenocaris dianae et Parastenocaris vandeli. Les 12 autres espèces, d'origine épigée, n'en sont pas moins des hôtes classiques des eaux souterraines (stygophiles). Au total, 29777 individus ont été récoltés.

\section{Analyse multidimensionnelle des données}

En raison du nombre élevé d'échantillons (130) et d'espèces (21), les données relatives aux Harpacticides ont été traitées par le biais de plusieurs analyses multidimensionnelles destinées à mettre en évidence les variations des échantillons par rapport soit au temps soit à l'espace.

\subsection{Evolution du peuplement par rapport au temps}

Afin de tester la présence éventuelle d'un cycle saisonnier, le temps a été introduit dans le tableau initial des données sous la forme d'une variable qualitative avec les modalités suivantes : 1 pour les 20 prélèvements de septembre 1985 ; 2 pour ceux de novembre $1985 ; \ldots 7$ pour les 10 prélèvements de septembre 1986.

Les espèces représentées par un seul ou deux individus ont été éliminées : en conséquence, 15 espèces seulement sur 21 ont été retenues.

Une analyse factorielle discriminante (AFD) a ensuite été effectuée sur le tableau ainsi modifié.

Les valeurs propres des axes discriminants sont faibles : 0,225 pour l'axe 1 (32,5\% de l'inertie) et 0,218 pour l'axe $2(31,5 \%$ de l'inertie) ce qui indique un pouvoir séparateur médiocre de la part de
Tableau 1. Liste des espèces d'Harpacticides et nombre d'individus récoltés.

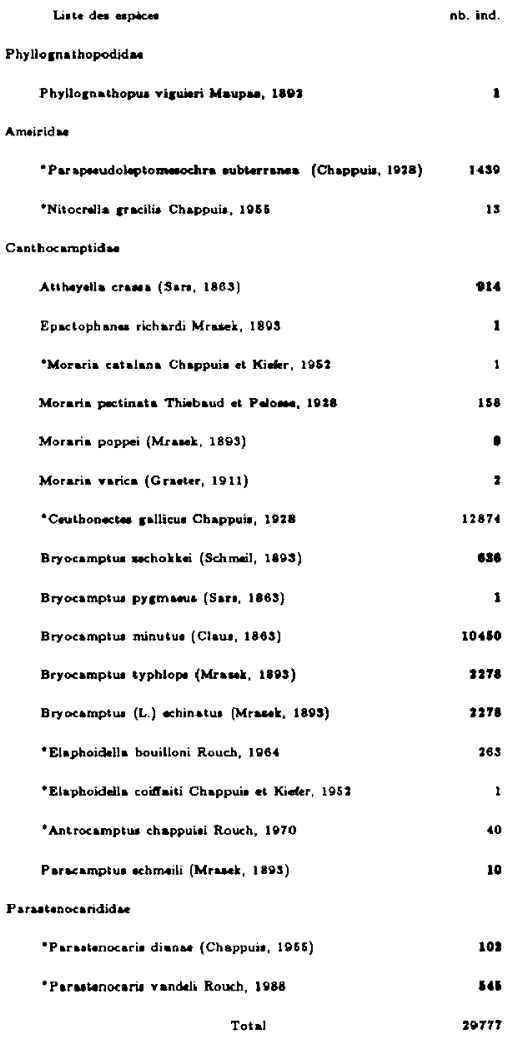

chacun de ces facteurs ; ce résultat est confirmé par les valeurs du pseudo $F(5,9$ et 5,7$)$ peu significatives.

Dans l'espace des variables (plan $F_{1}-F_{2}$ ), l'axe I est caractérisé par les espèces épigèes $A$. crassa, $M$. poppei et $P$. schmeili et l'axe 2 par les espèces hypogées $P$. vandeli, $N$. gracilis et l'espèce épigéc B. minuus (fig. 2). 


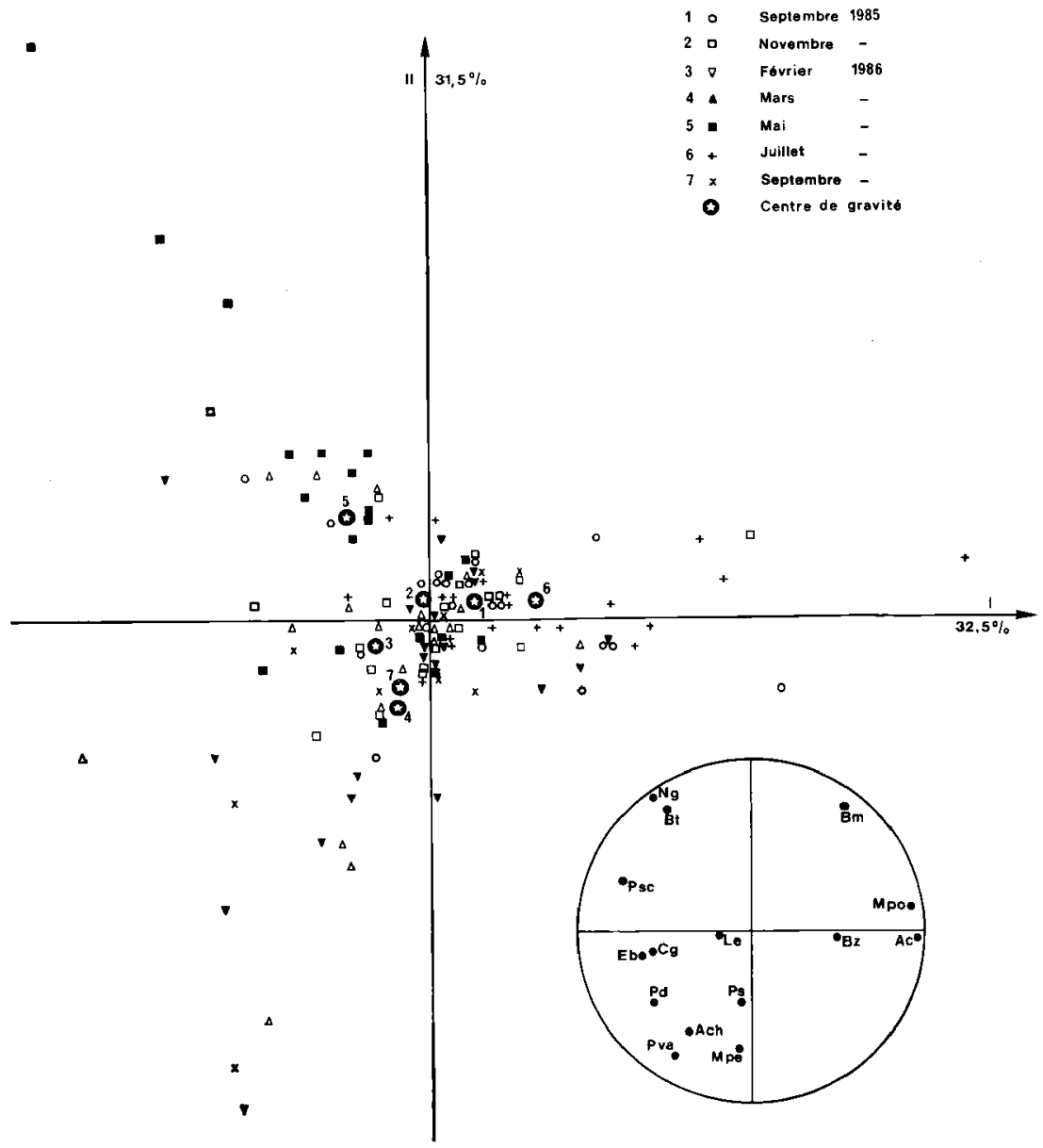

Fig. 2. Analyse factorielle discriminante des sept séries de prélèvements en fonction du temps. 
Dans l'espace des individus, les coordonnées des centres de gravité de chaque série de prélèvements sont proches du centre de gravité de la figure. Les valeurs des carrés des cosinus de ces centres de gravité sont inférieures à 0,5 sauf pour le groupement $6(0,917)$ sur l'axe 1 et pour le groupement $5(0,641)$ sur l'axe 2 . On peut en conclure que les sept séries de prélèvements sont mal définies par rapport au temps. Ce résultat est confirmé par la dispersion des échantillons dans chaque série de prélèvements. Deux tendances n'en sont pas moins apparentes ; les échantillons de juillet 1986 (groupe 6) sont, pour l'essentiel, rassemblés sur la partie positive de l'axe 1 en raison, notamment, de la capture de $M$. poppei à cette époque (4 individus sur les 8 récoltés au total). Les prélèvements de mai 1986 (groupe 5), caractérisés par la présence de $N$. gracilis (les 13 individus de cette espèce ont été capturés à cette date) sont en partie regroupés sur la partie positive de l'axe 2. Ces tendances, obtenues par l'apparition d'espèces rares, ne remet tent pas en cause l'absence de discrimination en relation avec le temps. Dans ces conditions, peu d'échantillons sont bien classés par rapport à la variable temps introduite dans l'analyse : seuls $31,5 \%$ d'entre eux sont affectés par l'analyse au groupe auquel ils appartiennent. Ainsi, au cours de l'année étudiée, le peuplement des Harpacticides ne présente pas de structure significative par rapport au temps. Ce résultat implique que les diverses populations d'Harpacticides n'ont pas subi d'évolution saisonnière.

\subsection{Structure du peuplement des Harpacticides dans l'espace}

L'hétérogénéité dont témoignent les échantillons ne pouvant être attribuée à une évolution saisonnière, pourrait correspondre à une organisation spatiale du peuplement. C'est ce qui a été recherché.

Dans un premier temps, les 130 échantillons prélevés ont fait l'objet d'une analyse factorielle des correspondances (AFC) pour laquelle n'ont été retenues que les 10 espèces constantes dans chaque série de prélèvements : Parapseudoleptomesochra subterranea, Ceuthonectes gallicus, Elaphoidella bouilloni, Parastenocaris dianae et Parastenocaris vandeli pour les espèces hypogées ; Bryocamptus minutus, Bryocamptus zschokkei, Bryocamptus (Limocamptus) echinatus, Bryocamptus typhlops et Attheyella crassa pour les espèces épigées (Rouch 1988). Ces espèces représentent $99,2 \%$ du total des Harpacticides capturés. Une classification automatique à partir des coordonnées des échantillons dans cette AFC conduit à répartir les prelèvements en quatre groupes, caractérisés chacun par l'abondance relative élevée d'une ou deux espèces.

Groupe 1: E. bouilloni $(40,9 \%)$; espèce hypogée.

Groupe $2: B(L$.$) echinatus (20,8 \%)$ et $A$. crassa $(24,5 \%)$; espèces épigées.

Groupe 3 : C. gallicus $(61,3 \%)$ et $P$. subterranea $(6,3 \%)$; espèces hypogées.

Groupe $4: B$. minutus $(67,3 \%)$; espèce épigée.

Ces différents groupes semblent correspondre à une organisation spatiale du peuplement : en effet, certains d'entre eux, notamment le 1 et le 3 , sont caractérisés par des espèces, E. bouilloni et $P$. subterranea, dont la répartition sur le site est extrêmement localisée (Rouch 1988). Toutefois 21 échantillons sur les 130 collectés présentent des caractères intermédiaires entre les quatre groupements observés.

Afin de préciser cette structure, une analyse factorielle discriminante a été réalisée où la variable qualitative introduite dans le tableau des données correspond aux quatre ensembles mis en évidence dans l'AFC. Les 109 échantillons bien classés précédemment ont été regroupés selon ces 4 modalités. Les 21 présentant des caractères intermédiaires ont été introduits sous forme d'unités supplémentaires. Comme dans l'analyse précédente, seules les 10 espèces constantes dans chaque série de prélèvements ont été retenues.

Les valeurs propres des axes discriminants sont élevées $(1,049-0,803-0,647)$ ce qui indique le fort pouvoir séparateur de ces axes, résultat confirmé par les valeurs importantes du pseudo $F$ (748 - 142 - 64). Le premier axe exprime $42,4 \%$ de l'inertie, le deuxième $32,4 \%$ et le troisième $26,1 \%$.

Dans le plan F1 - F2 de l'espace des variables (fig. 3), l'axe 1 sépare les espèces hypogées ( $C$. gallicus, $P$. subterranea et $P$. vandeli) des espèces épigées $B(L$.) echinatus et $A$. crassa. La partie positive de l'axe 2 est surtout caractérisée par l'espèce souterraine $E$. bouilloni et l'espèce épigée $B$. typhlops. 


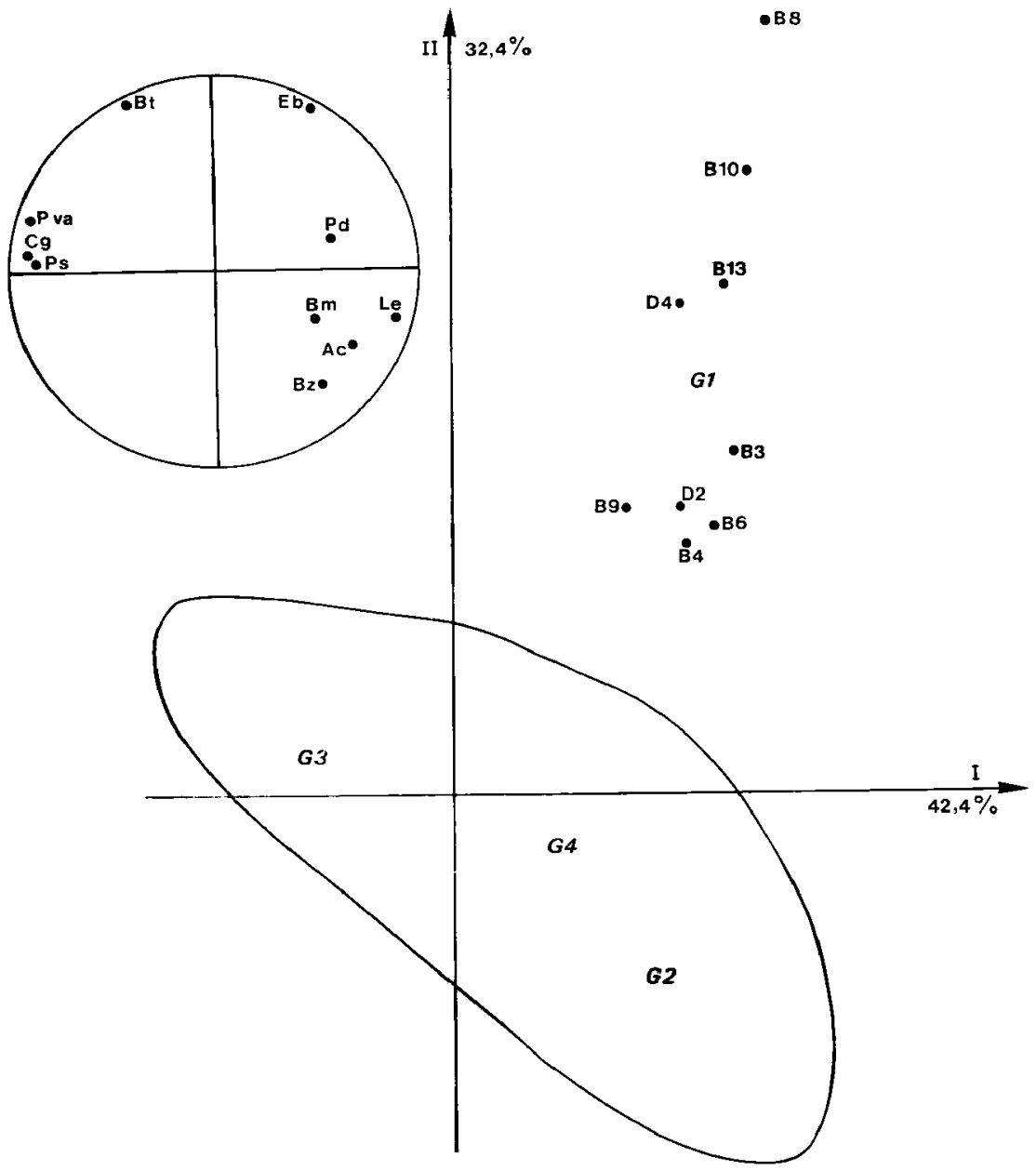

Fig. 3. Analyse factorielle discriminante en fonction de l'espace (facteurs 1 et 2 ). Seuls les prélèvements appartenant au groupe 1 ( $E$. bouilloni) ont été représentès. 
Dans l'espace des individus $\left(\mathrm{F}_{1}-\mathrm{F}_{2}\right)$, les centres de gravité du groupe 3 ( $C$. gallicus et $P$. subterranea) sur l'axe 1 et du groupe 1 ( $E$. bouilloni) sur l'axe 2 sont bien représentés (valeurs respectives des cosinus carrés 0,813 et 0,704 ) alors que les groupes 2 (B. (L.) echinatus et $A$. crassa) et 4 (B. minutus) sont moins nettement définis. Dans ce plan, l'axe 1 oppose les groupements dominés par les espèces épigées (G2 et G4) à celui marqué par les espèces hypogées $C$. gallicus et $P$. subterranea (G3). L'axe 2 différencie le groupe 1 (E. bouilloni) de tous les autres.

L'étude du plan F1 - F3 permet de préciser la partition des groupes 2,3 et 4 (fig. 4).

Dans l'espace des variables, comme on l'a vu, l'axe 1 oppose les espèces épigées $L$. echinatus et $A$. crassa (partie positive) aux espèces hypogées $C$. gallicus, $P$. subterranea et $P$. vandeli (partie négative). L'axe 3 est surtout déterminé, dans sa partie positive, par l'espèce épigée $B$. minutus et l'espèce hypogée $P$. dianae.

Dans l'espace des individus, l'axe 1 sépare le groupe 3 (hypogé) localisé dans sa partie négative, des groupes G2 et G4 (épigés) qui caractérisent sa partie positive. Les groupes G2 et G4 sont bien représentés sur l'axe 3 avec pour valeurs respectives des cosinus carrés 0,350 et 0,782 . La partition sur cet axe oppose le groupe 4 où domine B. minutus (partic positive) au groupe 2 déterminé par les espèces $L$. echinatus et $A$. crassa (partie négative).

Le groupe 1 ( $E$. bouilloni), défini par l'axe 2, est mal représenté dans cet espace.

Au total, parmi les 109 échantillons faisant l'objet de cette analyse, le pourcentage de ceux qui sont bien classés dans les quatre groupements initialement retenus est élevé puisqu'il atteint $99.1 \%$. Les 21 échantillons supplémentaires, classés selon leur distance par rapport aux centres de gravité des groupements, se répartissent à la périphérie des groupes 2,3 et 4 (voir figure 4 ).

Cette analyse confirme donc l'existence d'une structure du peuplement fondée sur l'appartenance écologique des espèces à deux grands groupes, celui des formes hypogées d'une part, celui des formes épigées d'autre part. Chacun de ces groupes est subdivisé en deux selon une partition qui intervient au niveau spécifique cette fois.

\subsection{Cartographie de la structure du peuplement des Harpacticides}

Comme chaque prélèvement a été repéré sur le site, il est possible de donner une représentation de la distribution spatiale des quatre groupements identifiés (fig, 5).

Le groupe 1, dominé par l'espèce $E$. bouilloni occupe l'aire réductrice, soit une partie des secteurs $B$ et $D$.

Le groupe 2, caractérisé par la fréquence élevée des espèces épigées $L$. echinatus et $A$. crassa, est cantonné au niveau du chenal qui s'étend le long de la rive droite (secteurs I, G, E et C en partie).

Le groupe 3, très fortement marqué par la dominance de $C$. gallicus et l'abondance relative de Parapseudoleptomesochra subterranea (espèces stygobies), s'étend sur les deux zones occupées par les bancs de graviers (secteurs $\mathbf{J}, \mathbf{H}, \mathbf{F}$ en rive gauche, secteur $A$ et une partie du secteur $C$ en rive droite).

Le groupe 4 où domine l'espèce épigée $B$. minutus occupe la périphérie des bancs de graviers.

Ainsi, globalement, les quatre groupements mis en évidence se superposent aux unités physiographiques définies par les écoulements de surface.

\section{Caractéristiques du peuplement des qua- tre zones}

\subsection{Richesse spécifique et densité}

S'il est tenu compte, maintenant, de la composition faunistique totale des quatre groupements définis par l'AFD (Tableau II), le fait est confirmé que la partition mise en évidence repose en grande partie sur des différences d'abondances relatives des espèces. L'exemple des groupes 2 et 4 , définis par des espèces épigées qui peuplent l'ensemble du site, est révélateur à cet égard. La composition taxonomique de ces deux groupes est identique. Tous deux ont pour espèce dominante $B$. minutus. C'est la fréquence relative de $L$. echinatus et $A$. crassa qui permet de les distinguer.

Toutefois, pour les groupes 1 et 3 , dominés par des formes hypogées, la discrimination observée repose sur des différences concernant aussi bien les fréquences des espèces communes aux deux groupes que leur composition faunistique. 


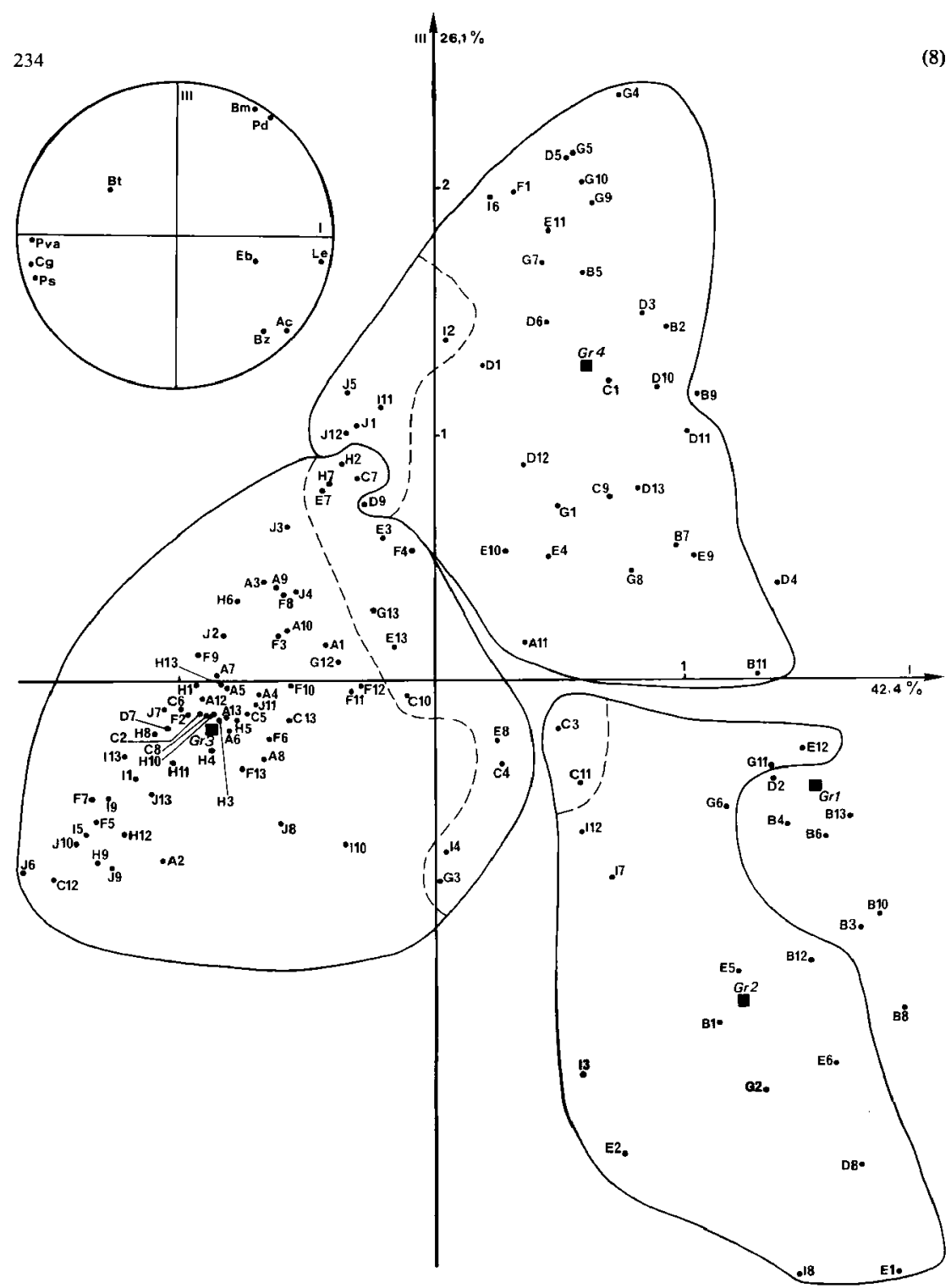

Fig. 4. Analyse factorielle discriminante en fonction de l'espace (facteurs 1 et 3). 
Tableau II. Composition faunistique et nombre d’individus récoltés dans les quatre groupes d'Harpacticides reconnus.

\begin{tabular}{|c|c|c|c|c|c|}
\hline Eapèces & G.1 & G.2 & G.3 & G.4 & $\mathbf{T}$ \\
\hline P.s & & 24 & 1188 & 227 & 1436 \\
\hline$N_{-g}$ & & & 13 & & is \\
\hline C.g & 1 & 280 & 11215 & 1378 & 12874 \\
\hline M.c & & & 1 & & 1 \\
\hline E.c & & & 1 & & 1 \\
\hline E.b & 255 & 1 & 1 & 6 & 263 \\
\hline A.ch & & & 10 & & 40 \\
\hline P.va & 4 & 5 & 487 & 49 & 545 \\
\hline P.d & 10 & 4 & 56 & 33 & 102 \\
\hline B.m & 220 & 560 & 4719 & 4951 & 10450 \\
\hline B.z & 16 & 58 & 370 & 192 & 696 \\
\hline B.P & 1 & & & & 1 \\
\hline B.t & 2 & 1 & 25 & 12 & 40 \\
\hline L.e & 79 & 438 & 911 & 850 & 2278 \\
\hline A.c & 61 & 385 & 313 & 155 & 914 \\
\hline E.r & & & 1 & & 1 \\
\hline M.pe & & 1 & 152 & 5 & 158 \\
\hline M.po & & 1 & 6 & 1 & 8 \\
\hline M.v & & & 2 & & 2 \\
\hline P.sc & & 8 & $\mathbf{3}$ & 5 & 10 \\
\hline P.v & 1 & & & & 1 \\
\hline & 650 & 1760 & & 7864 & \\
\hline
\end{tabular}

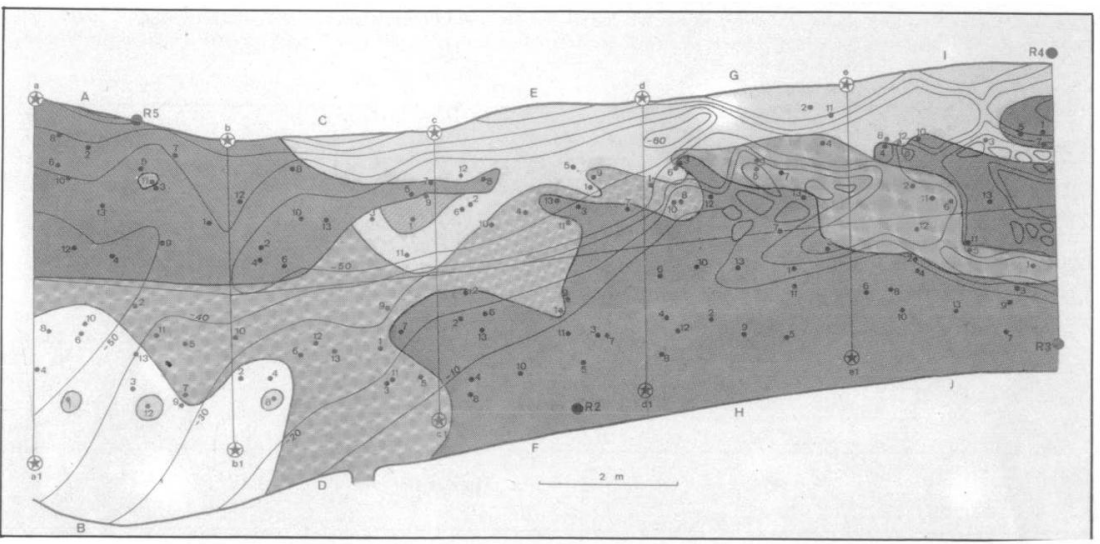

Fig. 5. Cartographie de la distribution des quatre groupes mis en évidence par l'analyse factorielle discriminante : en blanc, groupe $I(E$. boulloni) ; en gris clair, groupe $2(B$. (L.) echinatus et $A$. crassa); en gris moyen, groupe 4 ( $B$. minutus); en gris foncé, groupe 3 (C. gallicus et $P$. subterranea). 
Au total, en tenant compte des pourcentages en formes épigées et hypogées, des richesses spécifiques globales et moyennes ainsi que des densités (Tableau III), les quatre groupements peuvent être facilement distingués.

Sur l'aire occupée par le groupe 1, Elaphoidella bouilloni assure l'essentiel du contingent hypogé $(39,2 \%$ sur $41,5 \%)$. La richesse spécifique globale est de 11 espèces seulement. La richesse spécifique moyenne par prélèvement est de $6(\mathrm{x}=5,77 ; \mathrm{s}=$ 1,394). La densité moyenne des individus n'atteint que $7 \mathrm{i} / \mathrm{l}(\mathrm{x}=7,2 ; \mathrm{s}=3,934)$.

Sur l'aire relative au groupe 2 ( $L$. echinatus, $A$. crassa), les espèces hypogées ne représentent que $17,8 \%$ de l'ensemble des individus capturés. La richesse spécifique globale est de 13 espèces. La richesse spécifique moyenne est voisine de 5 ( $\mathrm{x}=$ $5,17 ; s=2,128)$. La densité moyenne des individus atteint $10 \mathrm{i} / \mathrm{l}(\mathrm{x}=10,3 ; \mathrm{s}=13,096)$.

Sur l'aire occupée par le groupe 3 (C. gallicus, $P$. subterranea), les espèces hypogées sont majoritaires $(66,6 \%)$. La richesse spécifique globale atteint 19 espèces. La richesse spécifique moyenne par prélèvement est égale à $7(\mathrm{x}=7,02 ; \mathrm{s}=1,239)$. C'est ici que la densité moyenne des individus est la plus élevée avec $28 \mathrm{i} / 1(\mathrm{x}=27,8 ; \mathrm{s}=13,744)$.
Dans l'aire correspondant au groupe 4 (B. minutus), les espèces hypogées ne représentent plus que $21,5 \%$ du total des individus récoltés. La richesse spécifique globale est de 13 espèces. La richesse spécifique moyenne par prélèvement est proche de 6 (x $=5,8 ; \mathrm{s}=1,532)$. La densité moyenne des individus atteint $23 \mathrm{i} / 1(\mathrm{x}=23,1 ; \mathrm{s}=25,093)$.

$\mathrm{Ce}$ sont les zones où les teneurs en $\mathrm{O}_{2}$ dissous (Rouch 1988) et les perméabilités (Rouch 1992) sont les moins élevées qui, globalement, présentent les peuplements les moins riches tant sur les plans quantitatif (densités) que qualitatif (richesse spécifique).

\subsection{Diversité spécifique}

Les valeurs des indices de diversité (Tableau III) des groupements provenant de la zone réductrice (1) et de sous le chenal (2) sont supérieures à celles des deux ensembles $(3,4)$ issus des bancs de graviers. Ce résultat est bien illustré par les courbes de dominance $\mathrm{k}$ (Lambshead et al. 1983) relatives à ces quatre groupements (fig. 6) : le 3 et le 4 sont caractérisés par une plus forte dominance de leur espece la plus abondante.

Le fait que les zones les moins favorables à l'implantation de populations abondantes d'Harpacticides possèdent les diversités Ies plus élevées pourrait paraître paradoxal. En effet, il est généralement

Tableau III. Caractéristiques des quatre groupements d'Harpacticides reconnus.

$\mathbf{n}=$ nombre de stations $; \mathbf{S}$ : richesse spécifique globale $; \mathrm{s}=$ richesse spécifique moyenne $; \mathbf{i} / \mathbf{l}=$ nombre d'individus par litre ;

1 - $\mathrm{D}=$ indice de diversité de Simpson $; \boldsymbol{H}=$ indice de diversite de Brillouin ; $\mathrm{H}^{\prime}=$ indice de diversité de Shannon-Wiener ; $\mathrm{d}$

$=$ fréquence de l'espèce la plus abondante.

\begin{tabular}{|lcccccccccc|}
\hline & $\mathrm{n}$ & \% hyp & \% epi & $\mathrm{S}$ &. & $\mathrm{i} / \mathrm{l}$ & $1-\mathrm{D}$ & $\mathrm{H}$ & $\mathrm{K}$ & $\mathrm{d}$ \\
\hline $\begin{array}{l}\text { G.1 } \\
\text { (E.b.) }\end{array}$ & 9 & 11,5 & 58,4 & 11 & 6 & 7,2 & 0,708 & 2,043 & 2,087 & 39,2 \\
\hline $\begin{array}{l}\text { G.2 } \\
\text { (L.e. et } \\
\text { A.c.) }\end{array}$ & 17 & 17,8 & 82,1 & 13 & 6 & 10,3 & 0,763 & 2,232 & 2,253 & 31,8 \\
\hline $\begin{array}{l}\text { G.3 } \\
\text { (C.g. et } \\
\text { P. s.) }\end{array}$ & 70 & 66,6 & $\mathbf{3 3 , 3}$ & 19 & 7 & 27,8 & 0,604 & 1,866 & 1,870 & 57,5 \\
\hline $\begin{array}{l}\text { G.4 } \\
\text { (B.m.) }\end{array}$ & 34 & 21,5 & 78,4 & 13 & 6 & 23,1 & 0,559 & 1,708 & 1,714 & 62,9 \\
\hline
\end{tabular}




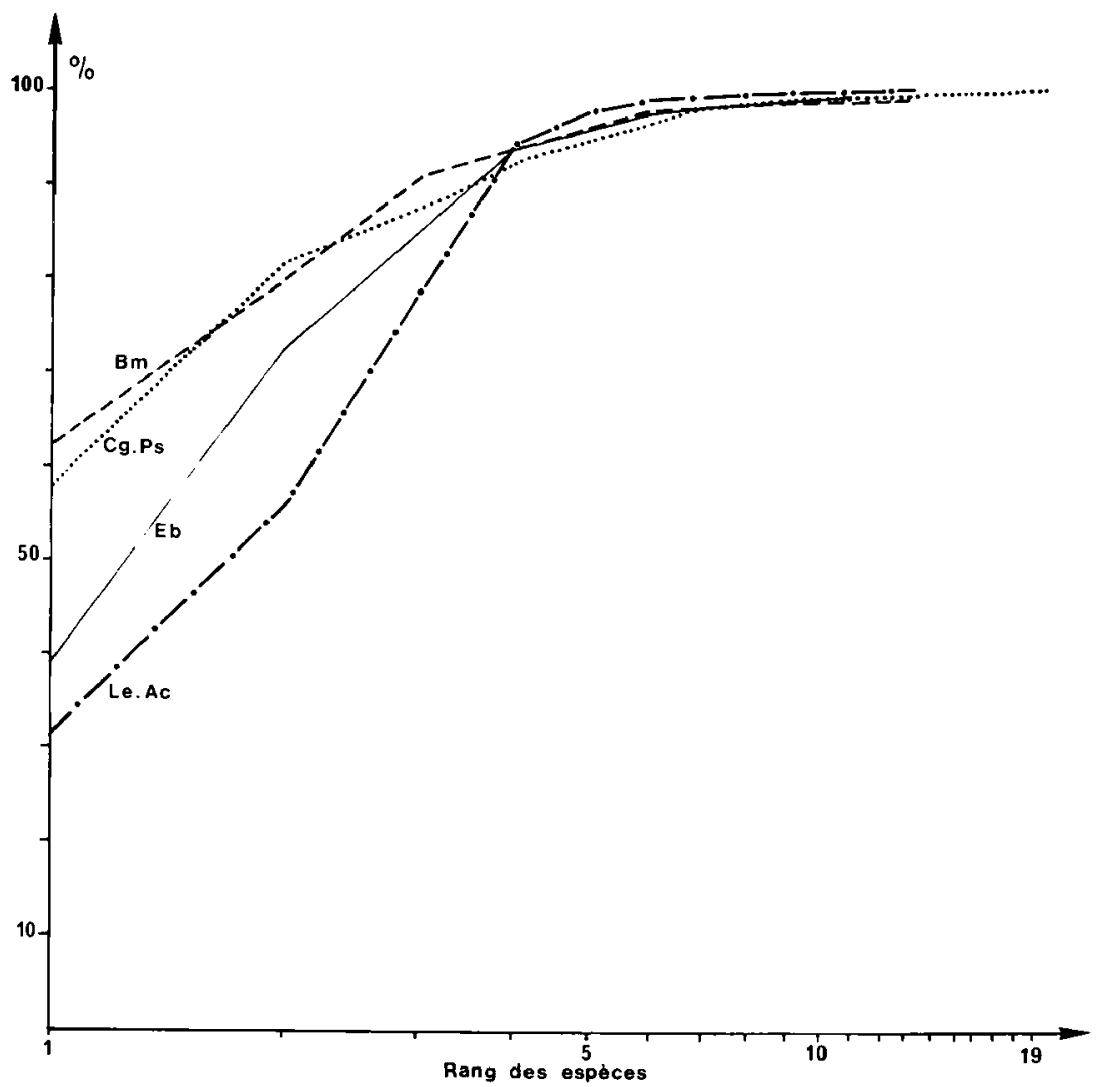

Fig. 6. Courbes de dominance-k des 4 groupes d'Harpacticides.

admis qu'un accroissement des contraintes de l'environnement se traduit par une diminution de la diversité.

Toutefois, dans son hypothèse générale sur la diversité spécifique, Huston (1979) suppose que la plupart des communautés naturelles ne sont pas à l'équilibre compétitif. Il propose alors un modèle d'équilibre dynamique qui prédit notamment que les communautés avec de faibles densités devraient avoir des diversités plus fortes que des communautés similaires où les densités sont plus élevées. Dans le premier cas, en effet, les interactions compétitives 
entre les espèces seraient faibles ce qui aurait pour effet de maintenir la diversité. Dans le second cas, au contraire, les phénomènes de compétition (exclusion compétitive) interviendraient qui diminueraient la diversité. Warwick \& Gee (1984), Platt \& Lamshead (1985), Lambshead (1986), dans une série d'exemples relatifs à la méiofaune marine, ont montré que sur des sites moyennement perturbés par des causes soit intrinsèques (conditions plus rigoureuses), soit extrinsèques (contamination), les valeurs des indices de diversité étaient plus élevées que celles provenant de sites non perturbés où des phénomènes d'exclusion compétitive peuvent intervenir.

Ces auteurs ont étayé leurs résultats en se fondant sur le modèle neutre de Caswell (1976) qui permet de générer une distribution des abondances relatives des espèces d'un prélèvement donné en excluant toute interaction biologique ou perturbation physique (Lambshead \& Platt 1988). Ce modèle permet de calculer une diversité $E\left(H^{\prime}\right)$ qui peut être comparée à la diversité réelle $H^{\prime}$ du prélèvement selon la formule :

$$
\begin{aligned}
V= & {\left[H^{\prime}-E\left(H^{\prime}\right)\right] / S D\left(H^{\prime}\right) \text { où } S D\left(H^{\prime}\right)=} \\
& \text { déviation standard de la diversité. }
\end{aligned}
$$

Quand V est proche de 0 , le prélèvement est considéré comme provenant d'un peuplement neutre. $\mathrm{V}>0$ indique un excès d'équitabilité et la diversité est plus grande que celle prédite. $\mathrm{V}<0$ traduit un excès de dominance et la diversité est inférieure à celle prédite. L'adéquation au modèle neutre peut être testée par la statistique F (Goldman \& Lambshead 1989).
Les données relatives aux quatre groupements du ruisseau de Lachein par rapport au modèle neutre de Caswell figurent dans le tableau IV.

Les groupes 1 et 2 ont une diversité $H^{\prime}$ supérieure à celle prédite par le modèle neutre $(V>0)$ alors que pour les groupes 3 et 4 , c'est l'inverse qui se produit $(V<0)$. On pourrait en conclure que dans les secteurs les plus contraignants, l'absence de compétition due à de faibles densités contribue à augmenter l'équitabilité. En revanche, dans les zones plus favorables où les densités sont élevées, des phénomènes d'exclusion compétitive interviendraient qui diminueraient la diversité. Cependant, cette interprétation doit être tempérée par le fait que la statistique $F$ présente des valeurs faibles qui n'impliquent pas une déviation significative de la neutralité.

\section{Discussion et conclusion}

Sur l'aire du ruisseau de Lachein étudiée, le peuplement des Harpacticides ne présente aucune variation saisonnière. Ce résultat est en accord avec la constance du rang des espèces observée tant au niveau de leur dominance que de leur fréquence (Rouch 1988). Kowarc (1991) signale également l'absence de cycle saisonnier dans les abondances des Harpacticides du Ritrodat (Autriche). En revanche, Pennak \& Ward (1984) remarquent que Phyllognathopus viguieri, l'espèce dominante dans le milieu hyporhéique de la South Platte River (Colorado) disparaît totalement de ce milieu au cours de la période hivernale. Strayer (1988) note aussi que les

Tableau IV. Modèle neutre de Caswell. H' : indice de Shannon-Wiener ; $\mathrm{E}\left(\mathrm{H}^{\prime}\right)$ : diversité prédite par le modèle neutre ; SD(H') : déviation standard de la diversité ; $V$ : déviation statistique ; F : statistique F. Les indices de diversité sont calculés en logarithmes népériens.

\begin{tabular}{llcccccr} 
& H' & $\mathrm{E}(\mathrm{H})^{\prime}$ & $\mathrm{SD}\left(\mathrm{H}^{\prime}\right)$ & $\mathrm{V}$ & $\mathrm{F}$ & \multicolumn{2}{c}{$\begin{array}{c}\text { degrés de liberté } \\
\text { de } \mathrm{F}\end{array}$} \\
$\mathbf{G . 1}$ & 1,45 & 1,42 & 0,306 & 0,08 & 1,05 & 16,4 & 11,3 \\
$\mathrm{G.2}$ & 1,56 & 1,44 & 0,321 & 0,38 & 1,22 & 16,5 & 12,9 \\
G.3 & 1,30 & 1,52 & 0,338 & $-0,66$ & 0,737 & 18,5 & 17,3 \\
G.4 & 1,19 & 1,27 & 0,349 & $-0,24$ & 0,875 & 12,4 & 12,6
\end{tabular}


Harpacticides subissent une évolution saisonnière dans le milieu hyporhéique de deux cours d'eau de l'Etat de New-York.

Les distributions des densités d'Harpacticides et des pourcentages en formes hypogées du milieu hyporhéique du ruisseau de Lachein ont conduit à reconnaître l'existence d'une structure spatiale de ce peuplement de Copépodes qui, globalement, se superpose aux différentes unitês physiographiques définies par les écoulements de surface (Rouch 1988). La mise en évidence de quatre groupements fondés sur les fréquences relatives des espèces constantes permet de préciser cette organisation spatiale.

Les sédiments sous le chenal et la zone réductrice offrent des conditions moins favorables à l'implantation de populations abondantes que ceux des bancs de graviers. Sous le chenal, la densité moyenne est peu élevée $(7 \mathrm{i} / \mathrm{l})$; les formes hypogées sont mal représentées $(18 \%)$, la richesse spécifique globale est de 13 espèces. Dans la zone réductrice, comme dans le chenal, la densité moyenne demeure faible $(10 \mathrm{i} / \mathrm{l})$; le pourcentage relativement élevé des individus hypogés $(41,5 \%)$ est dû, pour l'essentiel, à la présence de l'espèce sténotope Elaphoidella bouitloni ; la richesse spécifique globale est de 11 espèces. Les bancs de graviers représentent un habitat beaucoup plus favorable pour les Harpacticides. Les densités y sont, en moyenne, supérieures à $23 \mathrm{i} / 1$. Il existe cependant une ségrégation nette entre la périphérie de ces bancs dont la forme dominante est l'espèce épigée $B$. minutus $(62,9 \%)$ et leur centre où l'espèce hypogée $C$. gallicus $(57,5 \%)$ devient prépondérante. A la périphérie des bancs, la richesse spécifique globale est de 13 espèces; les formes hypogées ne représentent que $21,5 \%$ des individus récoltés. Au centre des bancs, la richesse globale atteint 19 espèces ; les individus hypogés constituent 66,6\% du peuplement. On notera toutefois que, en raison de la dominance élevée de $\boldsymbol{B}$. minutus et $C$. gallicus dans les bancs de graviers, les valeurs de la diversité y sont moins élevées que dans la zone réductrice ou sous le chenal, zones pourtant moins favorables.

L'hétérogénéité spatiale de la distribution des Harpacticides sur ce site est en accord avec les observations effectuées sur ce groupe par Whitman et Clark (1984) dans un cours d'eau du Texas et par Kowarc $(1987,1991)$ sur le Ritrodat. Dans cette station, les bancs de graviers constituent également l'habitat préférentiel des Harpacticides; mais, en l'absence d'espèces hypogées, les formes épigées occupent l'ensemble de ce biotope. Sur le ruisseau de Lachein, au contraire, les formes hypogées sont prépondérantes au centre des bancs de graviers. La distribution des densités des deux espèces les plus abondantes de ce peuplement est très significative à cet égard. La forme épigée $B$. minutus colonise l'ensemble du site (Rouch 1988). Sa densité moyenne est de $3,3 \mathrm{i} / 1$ sous le chenal et de $2,4 \mathrm{i} / \mathrm{I}$ dans l'aire réductrice ; elle atteint $14,5 \mathrm{i} / \mathrm{I}$ à la périphérie des bancs et retombe ensuite à $6,7 \mathrm{i} / 1$ en leur centre. L'espèce hypogée $C$. gallicus est pratiquement absente dans la zone réductrice : sa densité passe de 1,6 i/l sous le chenal à $4 \mathrm{j} / \mathrm{l}$ à la périphérie des bancs pour atteindre finalement $16 \mathrm{i} / 1$ au centre de ces bancs.

Ce résultat conforte l'opinion exprimée par Danielopol (1991) selon laquelle la faune hypogée peut non seulement vivre dans les sédiments superficiels mais aussi y surpasser les espèces épigées, témoignant ainsi d'une meilleure compétitivité.

Cet te hétérogénéité spatiale de la distribution des espèces et des individus se retrouve également chez un autre groupe de Crustacés microscopiques, les Ostracodes, dont Marmonier (1984, 1985) a montré que leur répartition se calque sur les différentes unités physiographiques du Ritrodat.

Sur le ruisseau de Lachein, l'étude de la granulométrie, de la porosité, de l'oxygène dissous (Rouch 1988), celle des caractéristiques chimiques de l'eau (Rouch et al. 1989) ont permis de montrer la singularité de chacune des zones façonnées par les écoulements de surface. Les fortes analogies observées entre la distribution de ces paramétres physicochimiques et celle du peuplement des Crustacés avaient conduit à reconnaître le rôle important joué par les écoulements de surface dans la structuration de ce milieu et de son peuplement.

L'étude de la surface pièzométrique et des valeurs de la perméabilité (Rouch 1992) dans ce secteur conduit à préciser ce point de vue.

Les bancs de graviers représentent une zone de drainage caractérisée par des valeurs de la perméabilité nettement plus élevées $\left(10^{-3} \mathrm{~m} / \mathrm{s}\right)$ que sous le chenal et l'aire réductrice $\left(10^{-4} \mathrm{~m} / \mathrm{s}\right)$, secteurs qui correspondent à des dômes piézométriques (fig. 7).

C'est au niveau de cette zone de drainage que les populations d'Harpacticides sont les plus abondantes et offrent la plus grande richesse spécifique. 

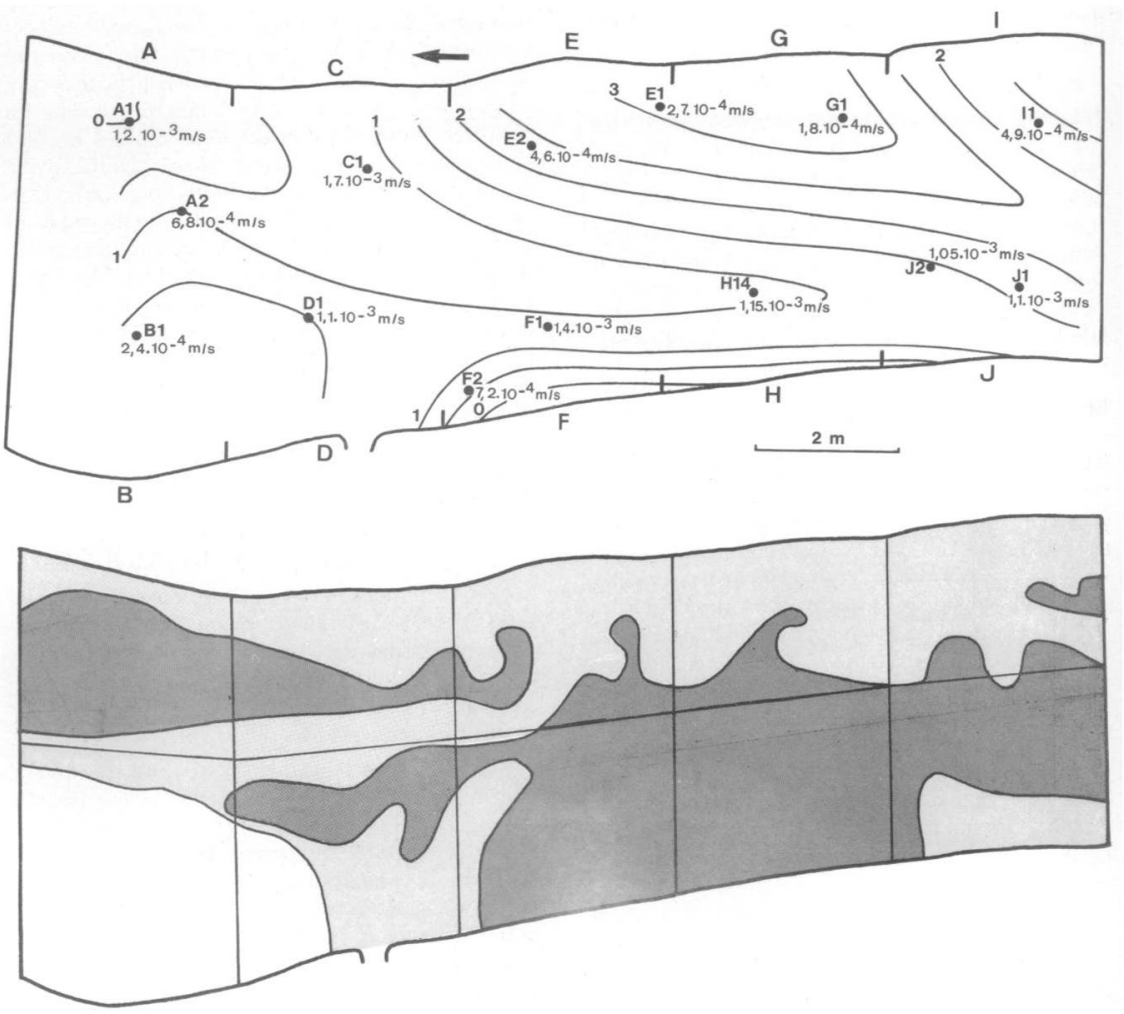

Fig. 7. En haut : carte de la surface piézométrique en étiage (espacement des courbes isopièzes $=0,5 \mathrm{~cm}$ ) et valeurs de la perméabilité (d'après Rouch 1992). En bas : répartition des facies géochimiques de l'eau interstitielle ; gris foncé : zone appauvrie en $\mathrm{CO}_{2}$ el bicarbonates ; gris clair : zone enrichie en silice et en sodium; blanc : zone réductrice, enrichie en $\mathrm{CO}_{2}$ et bicarbonates.

Il est donc évident que les conditions hydrodynamiques jouent un rôle fondamental dans l'hétérogénéité observée. Elles interviennent à deux niveaux.

Les écoulements de surface sont à l'origine des zones de dépôts et d'affouillement qui caractérisent la morphologie du secteur étudié ; ils contribuent de ce fait à déterminer les caractéristiques granulo- métriques et la porosité de ces différentes unités physiographiques.

Les conditions d'écoulement au sein des sédiments dépendent des valeurs de la perméabilité et des relations avec les nappes latérales. Ce sont ces conditions d'écoulements dans les sédiments qui permettent d'expliquer les partitions observées tant sur 
le plan chimique (fig. 7) que biologique (fig. 5) : la similitude de la distribution des caractéristiques chimiques de l'eau d'une part, des Harpacticides d'autre part, permet d'en mesurer l'importance.

Angelier (1962) avait pressenti ce double rôle des " courants superficiels et interstitiels » dont Godbout \& Hynes (1982), Marmonier \& Dole (1986), Dole-Olivier \& Marmonier (1992 a et b) ont souligné l'importance. Cette double action des processus géomorphologiques et hydrogéologiques est également prépondérante à l'échelle métrique, comme en témoigne cette étude. Creuzé des Châtelliers (1991) en a aussi montré toute la portée à l'échelle régionale.

\section{Remerciements}

Je remercie $A$. Descouens qui a participé aux prélèvements sur le terrain, trié la faune et réalisé les dessins définitifs de cette note ainsi que D. D'Hulst pour son aide concernant la partie informatique. Je remercie également P. J. D. Lambshead (The Natural History Museum, London) qui m'a trés aimablement communiqué le programme qu'il a mis au point pour le modèle neutre de Caswell. F. Boincau a saisi le manuscrit.

\section{Travaux cites}

Angelier (E.). 1962. - Remarques sur la répartition de la faune dans le milieu interstitiel hyporhéique. Zool. Anz, 168 : $351-356$.

Bou (C.) \& Rouch (R.). 1967. - Un nouveau champ de recherches sur la faune aquatique souterraine. C.R. Acad. Sci. Paris, $265: 369-370$

Caswell (H.). 1976. - Community structure : a neutral model analysis. Ecol, Monogr., 46 : 327-354.

Chappuis (P.A.). 1936. - Subterrane Harpacticoiden aus Jugoslavien. Bull. Soc. Sc. Cluj., $8: 368-398$.

Creuzé des Châtelliers (M.). 1991. - Dynamique de répartition des biocénoses interstitielles du Rhône en relation avec des caractéristiques géomorphologiques. Thèse doc. Univ. Lyon l, $161 \mathrm{p}$.

Daniclopol (D.). 1989. - Groundwater fauna associated with riverine aquifers. J.N. Am. benthol. Soc., $8:$ 18-35.

Danielopol (D.). 1991. - Spatial distribution and dispersal of interstitial Crustacea in alluvial sediments of a backwater of the Danube at Vienna. Stygologia, 6:97-110.

Dole-Olivier (M.J.) \& Marmonier (P.). 1992 a. - Patch distribution of interstitial communities : prevailing factors. Freshwater Biology, sous presse.

Dole-Olivier (M.J.) \& Marmonier (P.). 1992 b. - Ecological requirements of stygofauna in an active channel of the $R$ hône river. Stygologia, sous presse.

Godbout (L.) \& Hynes (H.B.N.). 1982. - The three dimensional distribution of the fauna in a single riffle in a stream in Ontario. Hydrobiologia, $97: 87-96$.

Goldman (N.) \& Lambshead (P.J.D.). 1989. - Optimization of the Ewens/Caswell neutral model program for community diversity analysis, Mar. Ecol. Prog. Ser., $50: 258-261$.

| Juston (M.). 1979. - A general hypothesis of species diversity. Am. Nat., $113: 81-101$.

Kowarc (V.). 1986. - Beiträge zur Kenntnis der Harpacticoidea im oberen Seebach. Jber. Biol. Sin. Lunz. 9 : 55-65.
Kowarc (V.) 1987. - Abundanzbestimmung und Populationsdynamik der Harpacticiden (Copepoda, Crustacea) im oberen Seebach. Jber. Biol. Sin. Lunz, 10 : 53-82.

Kowarc (V.). 1990 a. - Production of a harpacticoid copepod from the meiofaunal community of a second order mountain stream. Stygologia, $5: 25-32$.

Kowarc (V.). 1990 b. - Temporal and spatial distribution patterns and production of harpacticoids (Copepoda, Crustacea) in a second order mountain brook. Jber. Biol. Sin. Lunz. $13: 115-117$.

Kowarc (V.). 1991. - Distribution of harpacticoids in a second order mountain strearn (Ritrodat-Lunz study area, Austria). Verh. Internat. Verein Limnol, $24:$ 1930-1933.

Lambshead (P.J.D.). 1986. - Sub-catastrophic sewage and industrial waste contamination as revealed by marine nematode faunal analysis. Mar. Ecol. Prog. Ser., $29: 247.260$.

Lambshead (P.J.D.) \& Platt (H.M.). 1988. - Analysing disturbance with the Ewens/Caswell neutral model : theoritical review and practical assessment. Mar. Ecol. Prog. Ser., 43 : $3 !-4 !$.

Lambshead (P.J.D.), Platt (H.M.) \& Shaw (K.M.). 1983. - The detection of differences among assemblages of marine benthic species based on an assessment of dominance and diversity. J. nat. Hist., $17: 859-874$.

Marmonier (P.). 1984. - Vertical distribution and temporal evolution of the ostracod assemblage of the Seebach sediments (Lunz, Austria). Jber. Biol. Sin. Lunz., $7: 49-82$.

Marmonier (P.). 1985. - Répartition spatiale des Ostracodes dans les sédiments d'un ruisseau alpin (le Seebach, à Lumz, Autriche). Verh. Internat. Verein. Limnol., 22 ; 2053-2057.

Marmonier (P.). 1988. - Bjocénoses interstitieltes et circulation des eaux dans le sous-écoulement d'un chenal aménagé du Haut-Rhône français. Thèse doc. Univ. Lyon I, 2 tomes, 1-16! et $1-108$.

Marmonier (P.) \& Dole (M.J.). 1986, - Les Amphjpodes des sédiments d'un bras court-circuité du Rhône. Sciences de l'eau, 5: 461-486.

Pennak (R.W.) \& Ward (J.V.). 1986. - Interstitial faunal communities of the hyporheic and adjacent groundwater biotopes of a Colorado mountain stream. Arch. Hydrobiol./suppl. $74,3: 356-396$.

Platt (H.M.) \& Lambshead (P.J.D.). 1985. - Neutral model analysis of patterns of marine benthic species diversity. Mar. Ecol. Prog. Ser., 24 : 75-81.

Rouch (R.). 1986. - Les Harpacticides souterrains des eaux douces continentales. In : Stygofatena Mundi : 321-355. Edited by L. Botosaneanu. Brill, Leiden.

Rouch (R.). 1988. - Sur la répartition spatiale des Crustacés dans le sous-écoulement d'un ruisseau des Pyrénées. Annis. Limnol., 24 (3): 213-234.

Rouch (R.). 1992. - Caractéristiques et conditions hydrodynamiques des ćcoulements dans les sédiments d'un ruisseau des Pyrénées. Stygologia (sous presse).

Rouch (R.), Bakalowicz (M.), Mangin (A.) \& D'Hulst (D.). 1989. - Sur les caractéristiques chimiques du sous-écoulement d'un ruisseau des Pyrénées. Ann/s. Limnol., 25 (1) : 3-16.

Strayer (D.). 1988. - Crustaceans and mites (Acari) from hyporheic and other underground waters in southeastern NewYork. Srygologia, 4 : 192-207.

Warwick (R.M.) \& Gee (J.M.). 1984. - Community structure of estuarine meiobenthos. Mar. Ecol. Prog. Ser., 18 : 97-111.

Whitman (R.L.) \& Clark (W.J.). 1984. - Ecological studies of the sand-dwelling community of an East-Texas stream. Freshwat. Invertebr. Biol, 5 : 59-79. 